\title{
No man's land: Ischemic mitral regurgitation after primary percutaneous coronary intervention
}

\author{
Christina M. Vassileva, MD, ${ }^{\mathrm{a}}$ and Maurice Enriquez-Sarano, $\mathrm{MD}^{\mathrm{b}}$
}

Mitral regurgitation (MR) complicates the course of $13 \%$ to $50 \%$ of acute myocardial infarctions (MI). ${ }^{1}$ Postinfarction (ischemic) MR (IMR) portends a poor prognosis during long-term follow-up and has been identified as an independent predictor of heart failure (HF) and reduced long-term survival. ${ }^{2}$ Moreover, the severity of IMR is related to adverse outcomes in a graded manner; increasing IMR (none vs mild vs moderate to severe) is associated with decreased 1- and 5-year survival rates. ${ }^{2,3}$ In 1 study, which excluded patients who were older than 65 years, who had previous MI, multiple comorbidities, or severe congestive heart failure, $19 \%$ of patients had MR at 5 to 8 weeks after the event. ${ }^{4}$ Even in these young and relatively healthy patients after first MI, mortality at 15 months was 3 times higher for patients with IMR compared with those without IMR $(9.8 \%$ vs $3.2 \%)$. In addition, HF developed relatively rapidly, and by 15 months, $20 \%$ of patients with IMR had mild to moderate HF compared with $3 \%$ of patients without IMR. $^{4}$

Timely reperfusion is the primary goal of therapy for acute ST-elevation MI (STEMI). In the current era, this is best accomplished by percutaneous coronary intervention (PCI) when resources are available. In addition, PCI is increasingly performed in the setting of non-ST-elevation MI (NSTEMI). Although our focus on revascularization strategies has brought about progress in the field of cardiovascular medicine, we have paid less attention to other issues at hand, such as the assessment of left ventricular (LV) function or MR after MI. Thus, we have limited our ability to give full consideration to possible therapies that may alleviate the HF that occurs at a persistently high rate after MI. Although current guidelines recommend assessment of LV function in the setting of acute MI, preprocedural screening for the presence of IMR is not performed in most patients referred for primary PCI, unless it is secondary to papillary muscle rupture and is associated with

From the Division of Cardiothoracic Surgery, ${ }^{\text {a }}$ Southern Illinois University School of Medicine, Springfield, Ill; and Division of Cardiovascular Diseases, ${ }^{\mathrm{b}}$ Mayo Clinic, Rochester, Minn.

Disclosures: Authors have nothing to disclose with regard to commercial support. Received for publication April 4, 2013; accepted for publication April 9, 2013.

Address for reprints: Christina M. Vassileva, MD, Department of Cardiothoracic Surgery, Southern Illinois University School of Medicine, 701 N First St, Room D319, PO Box 19638, Springfield, IL 62794-9638 (E-mail: cvassileva@siumed. edu).

J Thorac Cardiovasc Surg 2013;146:2-3

$0022-5223 / \$ 36.00$

Copyright (c) 2013 by The American Association for Thoracic Surgery

http://dx.doi.org/10.1016/j.jtcvs.2013.04.004 major hemodynamic instability necessitating prompt surgical management.

Despite the poor prognosis with chronic IMR after primary PCI, there are no established guidelines for the routine follow-up of these patients. In fact, a recent survey of more than 1000 cardiovascular specialists from the United States and worldwide demonstrated that $25 \%$ did not even evaluate for the presence of IMR at the time of PCI. ${ }^{5}$ This percentage was as high as $32 \%$ in some regions of the United States. ${ }^{5}$ Improvement and even resolution of IMR may be expected after primary PCI in some cases. On the other hand, the absence of IMR at the initial presentation does not ensure freedom from IMR on longer-term follow-up because the post-MI LV remodeling may occur over several months or years. Unlike IMR in the coronary artery bypass graft population, little is known about the factors that may influence progression of IMR after primary PCI.

The most important strategy to ameliorate LV remodeling after MI remains early reperfusion. Theoretically, early reperfusion should halt the process of LV remodeling and the subsequent development of IMR. Indeed, there are some anecdotal reports from the 1980s of resolution of IMR with reperfusion alone. ${ }^{6,7}$ In general, successful reperfusion with primary PCI does not predictably result in improvement of IMR. ${ }^{8}$ In $43 \%$ of patients with IMR after first acute MI, IMR was still present 10 days later and was unrelated to the patency of the culprit vessel. ${ }^{7}$ Longer-term studies at 1,2, and 3 months, and up to 1 year, demonstrate that IMR persists in many patients. ${ }^{4,8}$

The most recent American College of Cardiology/American Heart Association focused update of the management of patients with valvular heart disease does not specifically address the subset of patients with IMR after primary PCI. ${ }^{9}$ This gap may be largely because of the paucity of data on these patients to allow the development of practice guidelines. We know that IMR is functional and is associated with poor outcome, but many uncertainties remain. We have not developed a uniform nomenclature of positive signs that allow us to link the MR to ischemic heart disease. Moreover, we have not uniformly accepted criteria of severity specific to IMR. We know that IMR may regress or progress, but we have not developed prevalence data in the chronic phase after MI and have not developed mechanistic factors that lead to the progression/regression of the IMR. Although IMR is a common complication of MI, the exact frequency with which it is detected largely depends on the modality used to look for its presence and the timing with 
respect to the acute MI. In earlier published studies, using angiography to document IMR, it was reported to occur in $13 \%$ to $19 \%$ of patients with acute MI. ${ }^{3}$ Subsequent studies using mainly color Doppler echocardiography report IMR with higher frequency, between $35 \%$ and $74 \%{ }^{1,2,8}$ The definition of chronic IMR with respect to the timing of the MI has also varied among studies. In the seminal work from the Survival and Ventricular Enlargement (SAVE) trial, Lamas and colleagues ${ }^{3}$ defined the early post-MI phase of IMR as fewer than 16 days after MI. Subsequently, Grigioni and colleagues ${ }^{2}$ coined the term chronic IMR to denote MR present for longer than 16 days after MI. Others have suggested that IMR may appear up to 6 weeks after MI as the LV undergoes remodeling.

The heterogeneity between study populations makes meaningful comparisons difficult. The available studies that include echocardiographic examination of patients after primary PCI can largely be divided into 2 subsets: (1) those that assess IMR early at some point after the MI event and subsequently provide long-term clinical follow-up and (2) those that provide serial echocardiographic data and correlate the presence and/or severity of IMR to clinical outcomes. Most studies fall into the first category, with echocardiographic examination provided within 30 days after the MI and no subsequent studies for comparison. ${ }^{1,6,7}$ Few studies report on long-term echocardiographic evaluation of patients after acute MI. ${ }^{2}$ These studies lack information on the presence and severity of IMR at the time of MI, but include a single echocardiographic assessment months to years after the index event and compare the clinical outcomes of patients with and without IMR. ${ }^{2}$ There are 2 serial echocardiographic studies in patients with acute MI undergoing PCI; in one, most patients were treated medically or underwent thrombolysis, and the second study was small and included only 2 serial echocardiographic examinations, one at the time of MI and the second 3 months after the event. $^{3,5}$

Despite the differences in study design and the heterogeneity of the patient populations, all reports consistently indicate that IMR after primary PCI portends a poor prognosis, with a reported 5 -year mortality of $40 \%$ to $55 \%{ }^{1}$ Yet, the most recent American College of Cardiology Foundation/American Heart Association updates of the guidelines on the management of patients with NSTEMI or STEMI do not address the issue of IMR, except for acute papillary muscle rupture. Furthermore, although evaluation of ventricular function is emphasized, the use of echocardiography is not required, provided left ventriculography is performed at the time of PCI. In a recent study of 5380 residents of the Worcester, Mass, metropolitan area who were admitted for acute MI between 1997 and 2005, 47\% of the patients did not receive echocardiography $(20 \%$ had an ejection fraction evaluation by left ventriculography, whereas the other $27 \%$ did not have an ejection fraction evaluation) in $2005 .^{10}$ Essential work remains to be done in terms of prevalence, mechanism, nomenclature, progression, outcome, and treatment of IMR. There appears to be an important need for the development of evidence-based guidelines for the follow-up of patients with acute MI treated with primary PCI, specifically addressing risk stratification with respect to IMR progression, and recommendations regarding optimal frequency of echocardiographic follow-up and timing mitral valve repair for IMR.

\section{References}

1. Bursi F, Enriquez-Sarano M, Nkomo VT, Jacobsen SJ, Weston SA, Meverden RA, et al. Heart failure and death after myocardial infarction in the community: the emerging role of mitral regurgitation. Circulation. 2005;111: 295-301.

2. Grigioni F, Enriquez-Sarano M, Zehr KJ, Bailey KR, Tajik AJ. Ischemic mitral regurgitation: long-term outcome and prognostic implications with quantitative Doppler assessment. Circulation. 2001;103:1759-64.

3. Lamas GA, Mitchell GF, Flaker GC, Smith SC Jr, Gersh BJ, Basta L, et al., Survival and Ventricular Enlargement Investigators. Clinical significance of mitra regurgitation after acute myocardial infarction. Circulation. 1997;96:827-33.

4. De Servi S, Vaccari L, Assandri J, Poma E, Cioffi P, Sciré A, et al. Clinical significance of mitral regurgitation in patients with recent myocardial infarction. Eur Heart J. 1988;9(suppl F):5-9.

5. Harris KM, Pastorius CA, Duval S, Harwood E, Henry TD, Carabello BA, et al. Practice variation among cardiovascular physicians in management of patients with mitral regurgitation. Am J Cardiol. 2009;103:255-61.

6. Heuser RR, Maddoux GL, Goss JE, Ramo BW, Raff GL, Shadoff N. Coronary angioplasty for acute mitral regurgitation due to myocardial infarction: a nonsurgical treatment preserving mitral valve integrity. Ann Intern Med. 1987;107: 852-5.

7. Lehmann KG, Francis CK, Dodge HT, TIMI Study Group. Mitral regurgitation in early myocardial infarction: incidence, clinical detection, and prognostic implications [published correction appears in Ann Intern Med. 1992;117:349]. Ann Intern Med. 1992;117:10-7.

8. Alam M, Thorstrand C, Rosenhamer G. Mitral regurgitation following first-time acute myocardial infarction: early and late findings by Doppler echocardiography. Clin Cardiol. 1993;16:30-4.

9. Bonow RO, Carabello BA, Chatterjee K, de Leon AC Jr, Faxon DP, Freed MD, et al. American College of Cardiology/American Heart Association Task Force on Practice Guidelines. 2008 Focused update incorporated into the ACC/AHA 2006 guidelines for the management of patients with valvular heart disease: a report of the American College of Cardiology/American Heart Association Task Force on Practice Guidelines (Writing Committee to revise the 1998 guidelines for the management of patients with valvular heart disease). Endorsed by the Society of Cardiovascular Anesthesiologists, Society for Cardiovascular Angiography and Interventions, and Society of Thoracic Surgeons. J Am Coll Cardiol. 2008;52:e1-142.

10. Joffe SW, Chalian A, Tighe DA, Aurigemma GP, Yarzebski J, Gore JM, et al Trends in the use of echocardiography and left ventriculography to assess left ventricular ejection fraction in patients hospitalized with acute myocardial infarction. Am Heart J. 2009;158:185-92. 